\title{
Uso de esteróides anabólicos androgênicos por praticantes de musculação de grandes academias da cidade de São Paulo
}

\author{
Luciana Silvia Maria Franco Silva, Regina Lúcia de Moraes Moreau*
}

Departamento de Análises Clínicas e Toxicológicas, Laboratório de Análises Toxicológicas da Faculdade de Ciências Farmacêuticas, Universidade de São Paulo.

*Correspondência:

R. L. M. Moreau

Departamento de Análises Clínicas e Toxicológicas

FCF-USP

Laboratório de Análises Toxicológicas Av. Prof. Lineu Prestes, 580, Bloco 13B 05508-900 - São Paulo, SP, Brasil E-mail: rlmoreau@usp.br
O objetivo desta pesquisa foi estimar o consumo e traçar o perfil dos usuários de esteróides anabólicos androgênicos (EAA) entre praticantes de musculação em três grandes academias de ginástica na cidade de São Paulo. Foi utilizado um questionário estruturado para ser respondido voluntária e anonimamente, com garantia explícita de confidencialidade para os mesmos. Os questionários ficaram disponiveis em três academias por uma semana, após ter sido feita ampla divulgação dos objetivos e importância do projeto. Responderam o questionário 209 praticantes de musculação (cerca de 3\% do total). A incidência de uso de EAA foi de 19\%, sendo que, destes, $8 \%$ declararam que fazem uso atualmente e $11 \%$, que já haviam feito uso anteriormente; considerando apenas o sexo masculino, a incidencia do uso foi de $24 \%$. Os compostos mais utilizados foram estanozolol e decanoato de nandrolona. O perfil dos usuários pôde ser delineado: idade média de 27 anos (de 25 a 29 anos), predominantemente homens, motivação pela melhora na estética corporal e treinamento muscular intenso. Os EAA foram adquiridos, em sua maioria, em farmácias, sem receita médica e foram feitos uso de suplemento alimentar e outros fármacos em associação. Acreditam que os efeitos tóxicos/adversos podem ser controlados elou evitados com o uso de outros medicamentos e/ou acompanhamento médico. O presente trabalho mostra a necessidade de investigações mais abrangentes e aprofundadas, bem como a adoção de ações preventivas e educativas junto à população exposta aos EAA.
Unitermos:

- Esteróides anabólicos androgênicos

- Praticantes de musculação

\section{INTRODUÇÃO}

Esteróides anabólicos androgênicos (EAA) sintéticos derivados da testosterona foram desenvolvidos com o propósito de se obter fármacos capazes de produzir aumento na síntese protéica (efeitos anabólicos) com menor grau de virilização (efeitos androgênicos). Centenas de compostos têm sido sintetizados, porém nenhum deles, até 
o momento, apresenta-se totalmente desprovido de atividade androgênica.

Frente aos inúmeros efeitos adversos indesejáveis que invariavelmente produzem, a utilização terapêutica destes compostos é rigidamente restrita a casos de hipogonadismo masculino, síndrome de Turner, tumor de mama, pré-menopausa, estados catabólicos graves e certos tipos de anemias refratárias a outras terapias (Mottram, George, 2000).

No meio esportivo, no início dos anos 50, fisiculturistas e halterofilistas foram os primeiros a utilizar EAA, com o objetivo de melhorar a estética corporal e o desempenho atlético. Este uso vem aumentando desde a década de 70, alastrando-se, também, entre indivíduos praticantes de outras modalidades esportivas, a despeito de serem consideradas substâncias de uso proibido pelo Comitê Olímpico Internacional (COI) e por Federações Desportivas Nacionais e Internacionais (Carlini-Cotrim, Barbosa, 1993; Mottram, George, 2000).

Atualmente, no panorama internacional, o consumo não médico dos EAA vem alcançando proporções alarmantes, atingindo outros segmentos da população, como freqüentadores de academias de ginástica e até estudantes de nível médio (Lindström et al., 1990; Perry et al., 1992; Evans, 1997; Korkia, Stimson, 1997; Lambert et al., 1998; Maharaj et al., 2000; Yesalis, Bahrke, 2000).

No Brasil, a prática da musculação tornou-se muito freqüente, principalmente entre adolescentes. Este padrão cultural reflete-se no número de academias de ginástica, que tem crescido vertiginosamente e se sofisticado, atraindo até investidores profissionais.

Na cidade de São Paulo, a primeira academia de ginástica com aulas de musculação conjuntas para homens e mulheres surgiu no início dos anos 80, com a introdução de exercícios aeróbicos. Atualmente, cerca de 3.500 academias estão implantadas na cidade, em um mercado que só tende a crescer.

Desde 1995, nas grandes academias de São Paulo, Rio de Janeiro e Brasília, o número de jovens entre 15 e 17 anos que praticam musculação tem triplicado. É fato notório, neste tipo de prática esportiva, a grande utilização de substâncias, como os EAA, destinadas a diminuir os depósitos de gordura e aumentar a força e massa muscular. No entanto, pouco ou nada se sabe acerca da incidência, prevalência e formas de uso dos diferentes produtos consumidos.

O uso disseminado de EAA é um problema extremamente sério, pois se trata de compostos de pronunciada toxicidade, utilizados indiscriminadamente e de maneira empírica (Maharaj et al., 2000; Mottram, George, 2000; Yesalis, Bahrke, 2000).
O objetivo desta pesquisa foi realizar estimativa do consumo e do comportamento dos usuários de EAA, praticantes de musculação, em três academias de grande porte, localizadas na cidade de São Paulo. Dados importantes para que se conheça este comportamento foram identificados: sexo, faixa etária, motivações para a prática de musculação, nível de treinamento muscular, uso de suplemento alimentar, identificação dos principais esteróides consumidos, formas de uso, produtos usados em associação e a maneira como os produtos foram obtidos. Também foram observados o aparecimento dos efeitos adversos e a atitude dos usuários frente ao conhecimento destes efeitos.

\section{MÉTODOS}

Praticantes de musculação de três grandes academias da capital de São Paulo foram escolhidos como população alvo. Um questionário sem identificação, em formato de fácil resposta, foi elaborado para que os participantes respondessem de modo voluntário. Um termo de compromisso anexado ao questionário assegurava o anonimato dos estabelecimentos e confidencialidade dos resultados. A permissão para a realização da pesquisa foi previamente obtida com o coordenador da academia. Após seu preenchimento, os questionários eram depositados pelo próprios voluntários em urnas lacradas, que ficaram disponíveis, por uma semana, em cada academia. A pesquisa foi previamente divulgada por meio de folhetos, cartazes e solicitação aos professores para que motivassem seus alunos a participar.

\section{RESULTADOS}

Dos cerca de 6.000 praticantes de musculação matriculados nas três academias, 209 (cerca de 3\% do total) responderam o questionário.

\section{Incidência de uso}

A incidência de usuários em geral foi de 19\% sendo que, destes, $8 \%$ declararam que estavam usando esteróides (usuários) e 11\% que já haviam feito uso anteriormente (exusuários).

\section{Sexo}

Dos indivíduos que responderam o questionário, cerca de $70 \%$ são do sexo masculino. Dentre a população total de usuários de EAA, 90\% são do sexo masculino. Considerando apenas os homens que responderam o questionário, $24 \%$ relatam ter feito uso de EAA considerando as mulheres, a incidência de usuárias é de 7\% (quatro usuárias). 


\section{Faixa etária}

O início do uso de esteróides é observado na população abaixo de 20 anos (5\%); este uso aumenta para $18 \%$ na faixa etária de 20 a 24 anos, atingindo a maior incidência na faixa de 25 a 29 anos (46\%). Após esta idade, o uso tende a declinar (13\%, tanto para a faixa de 30 a 34 anos, como na de 35 a 39 anos e $5 \%$ para acima de 40 anos).

\section{Motivações para prática de musculação e uso de esteróides}

O principal fator que induziu usuários e ex-usuários de esteróides a praticarem musculação foi a possibilidade de melhora na estética corporal (82\%), seguido da manutenção da saúde (41\%). Não usuários de EAA declararam ter essas mesmas motivações distribuídas entre 69 e $67 \%$, respectivamente.

A principal motivação para o uso de esteróides também foi a melhoria da aparência, com a mesma incidência de $82 \%$.

\section{Nível de treinamento muscular}

O nível de treinamento muscular é maior entre usuários em geral, tanto no aspecto do tempo de prática de musculação (para o período superior a dois anos, a incidência é $72 \%$ contra $28 \%$ de não usuários) quanto no aspecto de freqüência semanal (para a freqüência de 5 a 6 vezes por semana, a incidência é $67 \%$ contra $32 \%$ de não usuários).
Quando é feita a estratificação entre usuários e exusuários, o nível de treinamento é maior entre usuários do que ex-usuários. A maior incidência para usuários é de 6 vezes por semana (46\%) enquanto para ex-usuários é de 5 vezes semanais (36\%). Destaca-se, também, incidência significativa de $18 \%$ para 7 vezes semanais para usuários, contra $0 \%$ para ex-usuários.

\section{Uso de suplemento alimentar}

Usuários e ex-usuários de EAA (80\%) utilizam algum tipo de suplemento alimentar, principalmente proteína (55\%); a incidência para não usuários é 38\%. Considerando usuários, $100 \%$ fazem uso, enquanto que ex-usuários, $64 \%$.

\section{Esteróides utilizados e forma de uso}

Os EAA preferencialmente utilizados pela maioria dos usuários e ex-usuários, são estanozolol e decanoato de nandrolona. Alguns produtos são preferidos pelos usuários, como os ésteres de testosterona e oximetolona, enquanto que outros só foram relatados por eles, como metandrostenolona, oxandrolona, trembolona e mesterolona, só foram relatados pelos usuários. Preferencialmente os usuários (53\%) utilizam dois tipos de esteróides durante uma série (período de administração que varia de 6 a 12 semanas). Cinco ou mais compostos foram utilizados por $24 \%$ dos usuários, seguido de três tipos de EAA por $12 \%$ dos mesmos. A Tabela I apresenta os esteróides anabólicos utilizados por praticantes de musculação nas três academias paulistanas envolvidas no estudo.

TABELA I - Esteróides anabólicos androgênicos utilizados por praticantes de musculação em três grandes academias da capital de São Paulo (especialidade farmacêutica entre parênteses)

\begin{tabular}{|c|c|c|}
\hline Esteróide anabólico androgênico & $\begin{array}{c}\text { Usuários } \\
(\%)\end{array}$ & $\begin{array}{c}\text { Ex- usuários } \\
(\%)\end{array}$ \\
\hline 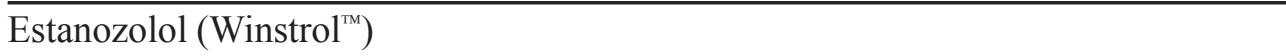 & 77 & 86 \\
\hline Decanoato de nandrolona (Deca-durabolin ${ }^{\circledR}$ ) & 76 & 55 \\
\hline Propionato, fenilpropionato, isocaproato e decanoato de testosterona (Durateston ${ }^{\circledR}$ ) & 71 & 32 \\
\hline Cipionato de testosterona (Deposteron ${ }^{\circledR}$ ) & 41 & 18 \\
\hline Oximetolona (Hemogenin ${ }^{\circledR}$ ) & 41 & 18 \\
\hline Undecanoato de testosterona (Androxon ${ }^{\circledR}$ ) & 41 & 14 \\
\hline Metenolona (Primobolan ${ }^{\mathrm{TM}}$ ) & 18 & 18 \\
\hline Enantato de testosterona (Testoviron ${ }^{\mathrm{TM}}$ ) & 6 & 5 \\
\hline Metandrostenolona $\left(\right.$ Dianabol $\left.^{\mathrm{TM}}\right)$ & 12 & 0 \\
\hline Oxandrolona $\left(\right.$ Anavar $\left.^{\mathrm{TM}}\right)$ & 12 & 0 \\
\hline Undecanoato de boldenona (Equipoise ${ }^{\mathrm{TM}}$ ) & 6 & 5 \\
\hline Trembolona (Parabolan ${ }^{\mathrm{TM}}$ ) & 6 & 0 \\
\hline Mesterolona (Proviron ${ }^{\circledR}$ ) & 6 & 0 \\
\hline Não responderam & 0 & 5 \\
\hline
\end{tabular}




\section{Produtos usados em associação}

No mínimo, $64 \%$ dos usuários e ex-usuários fazem uso de outros produtos em associação com EAA. Dentre eles, os mais citados foram efedrina e clembuterol. De maneira geral, usuários associam outros produtos com maior freqüência do que faziam os ex-usuários. A tendência dos usuários é utilizar principalmente efedrina e, em segundo lugar, clembuterol, inversamente em relação aos ex-usuários. Também utilizam com maior freqüência o tamoxifeno e outros produtos que não foram utilizados pelos ex-usuários. A Tabela II apresenta as principais classes de fármacos usados em associação com EAA.

\section{Acesso aos esteróides}

A maioria dos usuários e ex-usuários tem ou teve acesso aos EAA por meio de outro praticante de musculação. Porém, quando é feita a estratificação entre usuário e ex-usuário, percebe-se uma tendência: atualmente, o acesso está mais freqüente por meio da farmácia, sem receita médica. $\mathrm{O}$ ex-usuário obtinha preferencialmente por meio de outro praticante de musculação (Tabela III).

\section{Efeitos adversos observados}

Os efeitos mais observados relatados pelo total de usuários do sexo masculino foram: aumento da libido, alteração do humor, agressividade e o aparecimento de acne e ginecomastia (Tabela IV). No caso do sexo feminino, não houve quantificação confiável em virtude do número muito pequeno (apenas 4 mulheres). Todavia, todas relataram pelo menos alguns dos seguintes efeitos: atrofia das mamas, aumento do clitóris, aumento da quantidade de pêlos, engrossamento da voz ou rouquidão, irregularidades no ciclo menstrual, aumento da libido e aparecimento de acne.

\section{Atitude dos usuários e ex-usuários $\mathrm{X}$ efeitos adversos}

A maioria dos usuários e ex-usuários tem a crença de que os efeitos adversos podem ser prevenidos com o uso de outros produtos $(69 \%)$ e que o acompanhamento médico ajuda a prevenir doenças futuras $(72 \%)$.

\section{DISCUSSÃO}

O objetivo deste trabalho foi realizar uma primeira

TABELA II - Produtos usados em associação com os esteróides anabólicos androgênicos

\begin{tabular}{|c|c|c|}
\hline Fármacos usados em associação com esteróides anabólicos androgênicos & Usuários (\%) & Ex- usuários (\%) \\
\hline Efedrina & 47 & 32 \\
\hline Clembuterol & 29 & 36 \\
\hline Diuréticos & 24 & 18 \\
\hline Tamoxifeno-antagonista de estrogênio & 18 & 5 \\
\hline hGH - hormônio de crescimento & 13 & 0 \\
\hline hCG - gonadotrofina coriônica humana & 6 & 5 \\
\hline Insulina & 6 & 0 \\
\hline Hepato protetor & 6 & 0 \\
\hline Regularizador & 6 & 0 \\
\hline Xenadrine $^{\circledR}$ & 0 & 5 \\
\hline Orimeten $^{\circledR}$ & 0 & 5 \\
\hline Nenhum & 24 & 23 \\
\hline Não responderam & 6 & 18 \\
\hline
\end{tabular}

TABELA III - Meios pelos quais usuários e ex-usuários têm acesso aos esteróides anabólicos androgênicos

\begin{tabular}{lcc}
\hline Meios de acesso aos esteróides anabólicos androgênicos & Usuários (\%) & Ex- usuários (\%) \\
\hline Na farmácia, com receita médica & 12 & 9 \\
Na farmácia, sem receita médica & 65 & 23 \\
Em outros estabelecimentos comerciais & 12 & 32 \\
Com outros praticantes de musculação & 41 & 68 \\
Outros contatos & 29 & 27 \\
\hline
\end{tabular}


TABELA IV - Efeitos adversos decorrentes do uso de esteróides anabólicos androgênicos relatados pelo total de usuários do sexo masculino

\begin{tabular}{lc}
\hline Efeitos adversos & Total de usuários (\%) \\
\hline Aumento da libido & 63 \\
Alteração do humor & 54 \\
Agressividade & 49 \\
Acne & 46 \\
Ginecomastia & 37 \\
Atrofia dos testículos & 14 \\
Estrias & 11 \\
Diminuição da libido & 11 \\
\hline
\end{tabular}

estimativa do consumo e do comportamento de usuários e ex-usuários de EAA praticantes de musculação em academias, no sentido de servir como um sinalizador do problema. Não sendo, portanto, um levantamento epidemiológico, os dados obtidos não sofreram um modelamento estatístico.

Foram selecionadas para esta pesquisa academias de grande porte, ou seja, aquelas caracterizadas pela freqüência de, no mínimo, 2.000 praticantes de musculação. Este universo considerado é compatível com a estimativa proposta, em virtude da quantidade e heterogeneidade do universo amostral.

Questionários de auto-preenchimento necessitam, normalmente, de uma validação, ou seja, de uma comparação dos resultados obtidos com medidas externas já validadas do mesmo fenômeno. Esta validação não foi feita neste trabalho pois, para EAA, assim como para drogas de abuso, não existe um padrão-ouro (referência) para medir esta prática ilegal e cercada de preconceitos, já que o usuário se esforça para não deixar vestígios de seu consumo (Carlini-Cotrim, Barbosa, 1993).

Mesmo com o anonimato e confidencialidade assegurados, pode-se inferir que o valor encontrado de $19 \%$ do total de usuários de EAA seja subestimado, pela tendência destes indivíduos se sentirem mais inibidos para preencher o questionário do que os não usuários.

Estudos similares, abordando potenciais consumidores de esteróides anabólicos em populações heterogêneas, mostram prevalência variando de 2,9\% (Maharaj et al., 2000 ) a $38,8 \%$ (Perry et al., 1992). Quando o universo amostral é mais homogêneo, valores maiores, na média, são encontrados, tais como 38,4\% (Lindström et al., 1990) entre fisiculturistas homens.

Os resultados do presente estudo mostram que o sexo masculino predominou tanto na quantidade dos questionários respondidos (70\%) como na maior incidência do total de usuários $(90 \%)$ e na maior incidência destes dentre a po- pulação masculina $(24 \%$, contra $7 \%$ dentre a população feminina). Este achado é semelhante ao encontrado na literatura, principalmente pelo fato do usuário de esteróides ser, quase que exclusivamente, do sexo masculino.

Korkia e Stimson (1997) investigaram o uso de EAA em 21 academias na Grã-Bretanha e encontraram que cerca de $70 \%$ dos homens responderam o questionário que foi aplicado; a incidência total de usuários que haviam feito uso de esteróides foi $94 \%$ do sexo masculino e $9,1 \%$ da população masculina contra $2,3 \%$ da feminina. Evans (1997) aplicou um questionário apenas para usuários de esteróides, até completar o número de 100 e verificou que todos que responderam eram homens. Lindström e colaboradores (1990) aplicaram um questionário para fisiculturistas, respondido por $81 \%$ dos homens. As mulheres foram excluídas da análise estatística de sua pesquisa, pois somente 3 em 33 usaram esteróides, o que foi considerado número pouco significativo. O'Sullivan e colaboradores (2000) recrutaram a população em geral que fazia uso de esteróides para um estudo clínico-médico e dos 58 que responderam, todos eram homens.

Com relação à faixa etária, praticamente a metade dos usuários e ex-usuários tem de 25 a 29 anos. Outros pesquisadores também encontraram maior incidência dos usuários nesta faixa etária (Evans, 1997; Lindström et al., 1990).

No presente estudo é mostrado, de forma contundente, que o apelo à estética é a grande motivação para o consumo de EAA e também para a prática de musculação, entre os usuários em geral. Este achado comprova que o uso de EAA já está disseminado em outros segmentos da população, além do atlético em que o uso era motivado, principalmente, pela melhora do desempenho em competições esportivas.

Este fato é um problema preocupante, pois o padrão cultural de beleza masculina atual é ter força e um corpo com músculos bem volumosos e definidos, com teor mínimo de gordura. A prática da musculação vem ao encontro deste ideal; o ganho de força e de massa é importante para a auto-estima do jovem, facilitando sua aceitação junto ao grupo de amigos e impressionando o sexo oposto. Para muitos, a "malhação" é mais que modismo: é uma obsessão. Colocam em risco a saúde em busca de um corpo perfeito, exagerando nos exercícios físicos, o que pode levar a danos irreparáveis, uma vez que há um limite genético para o desenvolvimento muscular. Dentro deste cenário, a sedução para o uso de esteróides é enorme, pela esperança de que estes ajudem a esculpir o corpo dos sonhos em um tempo menor ainda do que aquele alcançado pela prática de musculação.

Apesar de resultados inconsistentes encontrados na literatura sobre a eficácia dos EAA, existe alguma evidência que o aumento de força e massa muscular pode estar 
maximizado com a administração de doses suprafisiológicas de esteróides em atletas previamente treinados, que estejam sendo submetidos a dietas hiperprotéicas e hipercalóricas e a treinamento intensivo com exercícios controlados (Bhasin et al., 2001).

Os resultados do presente estudo mostram que os usuários e ex-usuários têm noção sobre a eficácia dos EAA, uma vez que a maioria pratica musculação há mais de 2 anos e com maior freqüência semanal do que os não-usuários. Esta tendência também é verificada quando é feita a estratificação: usuários treinam mais vezes por semana do que ex-usuários. Também é marcante o maior consumo de suplementos alimentares, em especial proteínas, entre os usuários e ex-usuários e na totalidade dos usuários.

O padrão de beleza, explorado pela mídia e também pela proliferação crescente de academias de ginástica (que oferecem equipamentos cada vez mais sofisticados e atraentes para a prática da musculação), propicia o uso disseminado de esteróides. Outro fator que aumenta sua disponibilidade é a falta de controle na comercialização de EAA nas farmácias. Nesta pesquisa, o principal local de acesso dos usuários é na farmácia e sem apresentação de receita médica; ex-usuários relatam que obtiveram com outro praticante de musculação.

Entre os usuários em geral existe uma cultura de caráter não científico sobre a melhor maneira de usar os EAA conforme a finalidade pretendida. Informações podem ser obtidas em manuais ou transmitidas oralmente por outros usuários, que descrevem o resultado conseguido por experiência própria.

Neste contexto, de maneira geral, são feitas séries com os EAA: um ou mais esteróides são administrados por um período de 6 a 12 semanas. Atletas, geralmente, utilizam a auto-administração com mais de um tipo de EAA ao mesmo tempo; esta forma é conhecida como "empilhamento". Normalmente, 2 a 3 séries são feitas por ano.

Os resultados deste estudo confirmam esta prática: a maioria dos usuários e ex-usuários $(65 \%)$ faz de 2 a 3 séries por ano e cerca de $70 \%$ dos usuários utilizam mais do que um EAA; o mais popular é o estanozolol, seguido do decanoato de nandrolona e ésteres da testosterona. Estes resultados são similares aos encontrados na literatura (Perry et al., 1992; Evans, 1997; Korkia, Stimson, 1997; Iñigo et al., 2000; O’Sullivan et al., 2000).

Acrescente-se a este padrão de uso o fato de os usuários e ex-usuários de EAA terem, também, a tendência de associar grande variedade de outros fármacos com o objetivo de aumentar os efeitos desejáveis e/ou controlar os efeitos adversos. Os resultados desta pesquisa revelam que a maioria dos usuários em geral faz uso de outros produtos, sendo que a efedrina e o clembuterol foram os princípios fármacos mais citados. Estes são agentes simpatomiméticos usados na terapêutica para o tratamento de doenças das vias aéreas superiores (tratamento da asma, resfriados). No meio esportivo em geral, a efedrina é normalmente utilizada pelo seu efeito estimulante; na década de 1990, ganhou popularidade pela descoberta do efeito termogênico observado em indivíduos obesos. No entanto, não há estudos que provam que a efedrina poderia apresentar este efeito em atletas ou naqueles relativamente não-obesos. Na década de 1980 , muitos atletas começaram a utilizar o clembuterol como agente anabolizante, com base em pesquisas que mostraram que esta substância aumenta a massa muscular de animais de criação. Fato preocupante é o uso do hormônio de crescimento (hGH) e insulina pelos usuários, substâncias estas que não eram utilizadas pelos ex-usuários e que atualmente são consumidas devido às propriedades anabólicas que lhes são atribuídas. São utilizadas por pequena porcentagem de usuários, provavelmente devido ao alto custo destes produtos.

Também, 69\% dos usuários e ex-usuários acreditam que os efeitos adversos podem ser prevenidos com uso de outros produtos; os mais relatados foram os diuréticos para diminuir a retenção de fluidos e o tamoxifeno (agente antiestrogênico), contra a ginecomastia. Todos estes compostos acarretam efeitos tóxicos por si só.

Os relatos dos efeitos adversos são coerentes com os descritos na literatura (Evans, 1997; Lindström et al., 1990; Maharaj et al., 2000; O’Sullivan et al., 2000).

A crença de que doenças futuras possam ser prevenidas por meio de acompanhamento médico na administração é um indício de preocupação com os efeitos adversos. Embora não haja evidências experimentais que sustentem inteiramente esta crença, algumas abordagens possíveis, tais como o papel dos exames clínicos e laboratoriais como balizamento do risco à saúde, devido à exposição aos EAA, são importantes para que o usuário construa a sua argumentação, no sentido de maximizar os benefícios pretendidos e minimizar os riscos associados.

Estes resultados podem fornecer subsídios para que sejam tomadas medidas eficientes no sentido de mudar o perfil do consumo de EAA na nossa sociedade, seja pelo desenvolvimento de programas de prevenção e educação dirigidos à populações específicas, seja pela validação metodológica para que levantamentos análogos mais amplos possam ser feitos.

\section{ABSTRACT}

Use of anabolic-androgenic steroids among body builders in major gym centers in São Paulo, Brazil

To estimate the use of anabolic-androgenic steroids (AAS) among body builders of three - professionally equipped 
private gym in São Paulo, Brazil, body builders answered voluntary and anonimously a structured multiple itens questionnaire which was available for a week in these gym centers. The participants were informed in advance of the aim of the study. Of the 209 body builders attending (3\% of the total), 19\% had used AAS at some stage. The stratification of the results showed that $11 \%$ were composed of ex-user and 8\% were of active users. Considering only male body builder the incidence of AAS was 24\%. Stanozolol and nandrolone decanoate were the most commonly AAS used. The significant features of their profile were: the median age of users was 27 (25-29) years, predominantely males and motivation for using AAS was aesthetic with a high level of physical training. Food suplements as well as other drugs in association were used. Mainly AAS were bought in drugstores without prescriptions. Body builders considerered anabolicandrogenic steroids to be dangerous and that the adverse effects can be managed or avoided with other drugs or medical care. This study demonstrate the use of AAS among body builders and consequently the necessity of deep preventive and educationaly investigation among people exposed to AAS.

UNITERMS: Anabolic-androgenic steroids. Body builders. User's profile.

\section{AGRADECIMENTO}

Os autores agradecem o apoio do Programa Institucional de Bolsa de Iniciação Científica (PIBIC), às Academias que permitiram o acesso aos praticantes de musculação, aos coordenadores de musculação, aos professores e a todos aqueles que, anonimamente, contribuíram para este levantamento, por meio do preenchimento do questionário.

\section{REFERÊNCIAS BIBLIOGRÁFICAS}

BHASIN, S.; WOODHOUSE, L.; STORER, T. W. Proof of the effect of testosterone on sketletal muscle. $J$. Endocrinol., Bristol, v.170, p.27-38, 2001.

CARLINI-COTRIM, B.; BARBOSA, M. T. Pesquisa epidemiológica sobre o uso de drogas entre estudantes: um manual de orientações gerais. São Paulo: CEBRID, Escola Paulista de Medicina, 1993. 56p.

EVANS, N. A. Gym and tonic: a profile of 100 male steroid users. Br. J. Sports Med., Loughborough, v.31, p.54-58, 1997.
IÑIGO, M. Á.; ARRIMADAS, E.; ARROYO, D. Estudio de 43 ciclos de tratamiento con anabolizantes esteroideos en deportistas: usos y efectos secundarios. Rev. Clin. Esp., Madrid, v.200, p.133-138, 2000.

KORKIA, P.; STIMSON, G. V. Indications of prevalence, practice and effects of anabolic steroid use in Great Britain. Int. J. Sports Med., Stuttgart, v.18, p.557-562, 1997.

LAMBERT, M. I.; TITLESTAD, S. D.; SCHWELLNUS, M. P. Prevalence of androgenic-anabolic steroid use in adolescents in two regions of South Africa. S. Afr. Med. J., Cape Town, v.88, p.876-880, 1998.

LINDSTRÖM, M.; NILSSON, A. L.; KATZMAN, P. L.; JANZON, L.; DYMLING, J. F. Use of anabolicandrogenic steroids among body builders: frequency and attitudes. J. Intern. Med., Oxford, v.227, p.407-411, 1990.

MAHARAJ, V. R.; DOOKIE, T.; MOHAMMED, S.; INCE, S.; MARSANG, B. L.; RAMBOCAS, N.; CHIN, M.; MCDOUGALL, L.; TEELUCKSINGH, S. Knowledge, attitudes and practices of anabolic steroid usage among gym users in Trinidad. West Indian Med. J., Kingston, v.49, p.55-58, 2000.

MOTTRAM, D. R.; GEORGE, A. J. Anabolic steroids. Bailliere's Clin. Endocrinol. Metab., London, v.14, p.5569, 2000.

O’SULLIVAN, A.; KENNEDY, M. C.; CASEY, J. H.; DAY, R. O.; CORRIGAN, B.; WODAK, A. Anabolicandrogenic steroids: medical assessment of present, past and potential users. Med. J. Aust., Glebe, v.173, p.323$327,2000$.

PERRY, H. M.; WRIGHT, D.; LITTLEPAGE, N. C. Dying to be big: a review of anabolic steroid use. Br. J. Sports Med., Loughborough, v.26, p.259-261, 1992.

YESALIS, C. E.; BAHRKE, M. S. Doping among adolescent athletes. Bailliere's Clin. Endocrinol. Metab., London, v.14, p.25-35, 2000.

Recebido para publicação em 12 de março de 2003. 